\title{
Ionospheric response to traveling convection twin vortices
}

\author{
R. W. Schunk, L. Zhu, and J. J. Sojka \\ Center for Atmospheric and Space Sciences, Utah State University, Logan, Utah 84322-4405
}

Abstract. Traveling convection twin vortices have been observed for several years. At ionospheric altitudes, the twin vortices correspond to spatially localized, transient structures embedded in a large-scale background convection pattern. The convection vortices are typically observed in the morning and evening regions. They are aligned predominantly in the eastwest direction and have a horizontal extent of from 500-1000 $\mathrm{km}$. Associated with the twin vortices are enhanced electric fields, particle precipitation, and an upward/downward fieldaligned current pair. Once formed, the twin vortex structures propagate in the tailward direction at speeds of several $\mathrm{km} / \mathrm{s}$, but they weaken as they propagate and only last for about 10 20 minutes. Because these convection structures might have a significant effect on the localized ionosphere, the USU ionospheric model was used to calculate the response of the ionosphere to "representative" traveling convection twin vortices for a range of background conditions. The ionospheric response includes localized temperature enhancements, ion composition changes, non-Maxwellian ion distributions, and plasma upwelling events. The response is transient and the magnitude of the response depends on the background ionospheric conditions and on the characteristics of the twin vortices.

\section{Introduction}

Ground-based magnetometers have observed the signatures of traveling convection twin vortices [Friis-Christensen et al., 1988; Heikkila et al., 1989; McHenry et al., 1990a, b; Kivelson and Southward, 1991; Glassmeier and Heppner, 1992]. The twin vortices correspond to spatially localized, transient, convection cells embedded in a large-scale convection pattern. The vortices are characterized by enhanced electric fields, particle precipitation, and an upward/downward field-aligned current pair. They are typically observed in the prenoon and postnoon sectors at geomagnetic latitudes between $60-75^{\circ}$ and are aligned predominantly in the east-west direction. The E-W extent of a vortex structure ranges from several 100 's to $1000 \mathrm{~km}$ and its N-S extent is about $500 \mathrm{~km}$. Although the field-aligned currents are opposite in a given twin vortex, either polarity is possible. The magnitude of the current is estimated to be about $1 \mu \mathrm{A} / \mathrm{m}^{2}$ and the characteristic energy of the precipitation in the upward current cell is about $1 \mathrm{keV}$. Once formed, the twin vortices propagate toward the nightside at speeds of from $3-6 \mathrm{~km} / \mathrm{s}$, but they weaken as they propagate and only last for about 10-20 minutes. Although single vortex structures are more common, continuous series of traveling twin vortices was observed to

\section{Copyright 1994 by the American Geophysical Union.}

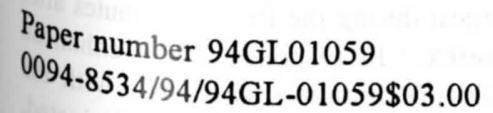

move in the antisunward direction for a period of several hours, with about a 15-minute time interval between vortices.

Mounting evidence indicates that the twin vortices occur on closed magnetic field lines and most likely those field lines that connect to the low-latitude boundary layers (LLBL) [Heikkila et al., 1989; McHenry et al., 1990a]. At present, the prevailing view is that the twin vortices are not a result of flux transfer events at the dayside magnetopause, but their exact cause is still controversial. Friis-Christensen et al. [1988] suggested that the traveling vortices are due to sudden changes in the solar wind dynamic pressure or the interplanetary magnetic field (IMF). Heikkila et al. [1989] suggested that the twin vortices are due to penetrating solar wind plasma clouds, while McHenry et al. [1990b] proposed that the vortices are due to a K-H instability at the LLBL. Unfortunately, to date, very little work has been done with regard to quantitative electrodynamic modeling of traveling convection twin vortices, although conceptual models of vortex generation have been developed that are based on the transient response of the magnetosphere to sudden solar wind dynamic pressure changes [Sibeck, 1990; Kivelson and Southward, 1991].

Our interest is not in modeling the traveling vortex structure, but in calculating the ionospheric response to such a structure. Therefore, any electrodynamic model that produces self-consistent electric field and particle precipitation patterns that are consistent with the measurements is adequate for our purposes. To this end, we used the electrodynamic model developed by Zhu et al. [1993] to obtain a "representative" traveling convection twin vortex. The ionospheric response to this vortex structure was then calculated with the USU ionospheric model [Schunk, 1988; Sojka, 1989].

\section{Ionospheric Model}

The USU time-dependent ionospheric model (TDIM) is a multi-species $\left(\mathrm{NO}^{+}, \mathrm{O}_{2}^{+}, \mathrm{N}_{2}^{+}, \mathrm{O}^{+}, \mathrm{N}^{+}, \mathrm{He}^{+}\right)$model that is based on a numerical solution of the coupled continuity, momentum, and energy equations. The TDIM is a Lagrange-Euler hybrid model in that the equations are solved as a function of altitude on a fixed grid for horizontally convecting plasma flux tubes. The 3-dimensional nature of the model is obtained by following numerous flux tubes in a given simulation. However, the TDIM requires several global inputs, with the primary ones being the atmospheric parameters and the magnetospheric convection and precipitation patterns.

The MSIS atmospheric model was adopted for the simulations, and both summer (day 173) and winter (day 357) cases were considered for low magnetic activity $\left(A p=9, K_{p}=\right.$ $2+)$ and moderate solar activity $\left(F_{10.7}=130\right)$. The neutral wind was described by our previously used simple prescription with a $100 \mathrm{~m} / \mathrm{s} \mathrm{N}-\mathrm{S}$ component. With regard to the magnetospheric inputs, both "background" and "twin vortex" convection and precipitation patterns were needed. Our selection of the background patterns was guided by observations, which 
indicated that twin vortices favor positive IMF $B$, conditions and relatively quiet geomagnetic activity [McHenry et al., $1990 \mathrm{~b}]$. Therefore, the background patterns we adopted were the Heppner-Maynard "distorted DE" convection pattern for northward $B_{z}$ [Heppner and Maynard, 1987] and the Hardy et al. [1985] auroral oval for $K_{p}=2+$. Note that different background convection and precipitation patterns could have been adopted, which would yield different background ionospheric conditions. However, the ionospheric response to a traveling twin vortex would be qualitatively similar.

The convection and precipitation signatures associated with the traveling twin vortex were obtained from the Zhu et al. [1993] electrodynamic model using several simplifying assumptions. First, it was assumed that a moving field-aligned current pair was generated owing to the interaction of the solar wind with the LLBL. The current filaments were initiated in the dawn sector, and then they were assumed to move toward the nightside at a speed of $3 \mathrm{~km} / \mathrm{s}$. A Gaussian was assumed to represent the current distribution in each filament, with a maximum current of $1.2 \mu \mathrm{A} / \mathrm{m}^{2}$ for both the upward and downward current filaments initially. As the twin vortex moved toward the nightside, the currents were assumed to decay in time. The field-aligned current filaments were sustained by a downward propagating Alfvén wave and, coupled with an ionospheric boundary condition, this allowed us to calculate self-consistent convection and precipitation patterns for a traveling twin vortex. However, for the calculation of the precipitation pattern, we made the additional assumption that only the upward current filament was associated with precipitating electrons. The resulting energy flux distribution was calculated according to Fridman and Lemaire [1980].

Before presenting the vortex patterns, it is useful to introduce the high-resolution spatial grid used in our simulations (Figure 1). This grid is located in the dawn sector of the polar region and is large enough to contain the traveling vortex during its lifetime. Snapshots of the electrostatic potential distribution that is associated with the traveling twin vortex are shown in Figure 2. The vortex was initiated at 12 UT in the high-resolution grid on the dayside. The potential distribution represents twin convection cells with the plasma convection counterclockwise in the nightside cell and clockwise in the dayside cell. Initially, the maximum convection electric field is about $100 \mathrm{mV} / \mathrm{m}$ (initial circulation speeds of about $2 \mathrm{~km} / \mathrm{s}$ ), but as the twin vortex structure moves

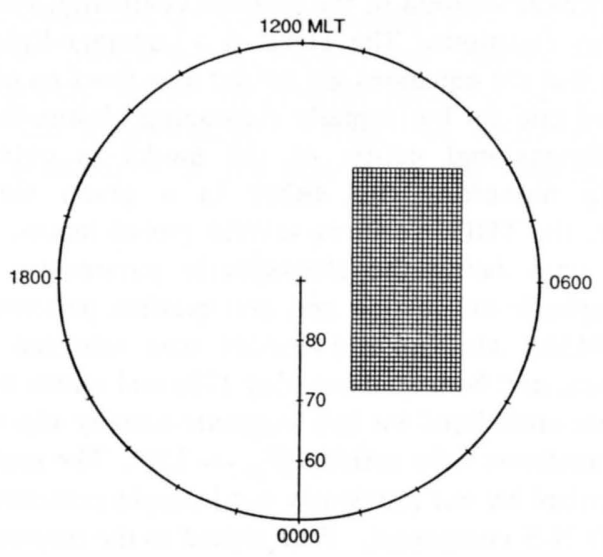

Figure 1. High-resolution spatial grid used in the simulations. The grid is shown in an MLT-magnetic latitude coordinate system and is approximately $2000 \times 4000 \mathrm{~km}$.
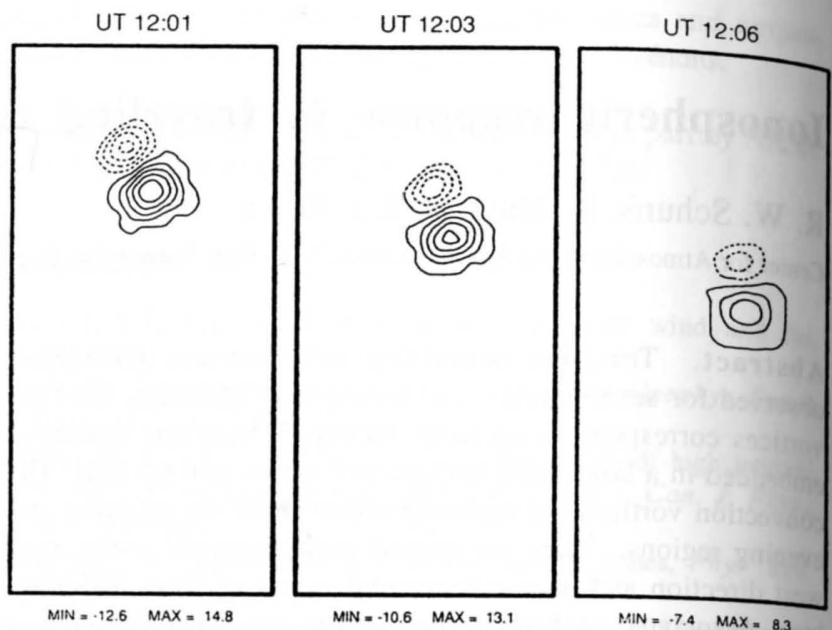

Figure 2. Snapshots of the potential distribution associated with the twin vortex. The contour spacing is $3 \mathrm{kV}$. The maximum and minimum potentials are 14.8 and $-12.6 \mathrm{kV}$ (left), 13.1 and $-10.6 \mathrm{kV}$ (middle), and 8.3 and $-7.4 \mathrm{kV}$ (right).

toward the nightside at a speed of $3 \mathrm{~km} / \mathrm{s}$, its intensity decreases and it disappears after approximately 15 minutes. The nightside and dayside cells also correspond to downward and upward field-aligned currents, respectively. Precipitating electrons only occur in association with the upward current (dayside cell), as explained earlier (not shown). Initially, the maximum precipitating energy flux is $2.3 \mathrm{ergs} \mathrm{cm}^{-2} \mathrm{~s}^{-1}$.

Note that the "representative" traveling twin vortex shown in Figure 2 has features that are both qualitatively and quantitatively in agreement with the observations. Therefore, these self-consistent inputs can be used in the TDIM to calculate the ionospheric response to a traveling twin vortex.

\section{Ionospheric Simulation}

The USU TDIM was used to calculate the ionospheric response to the traveling twin vortex. The ionospheric parameters were obtained at altitudes from 90 to $800 \mathrm{~km}$ with a $4 \mathrm{~km}$ spatial step. Approximately 1000 plasma flux tubes were followed, with the result that one flux tube always existed in each of the grid cells shown in Figure 1 at every time step. This yielded a $1 \mathrm{~km}$ horizontal spatial resolution. The plasma flux tubes were interpolated to the grid locations to obtain the magnetospheric inputs associated with the twin vortex. The time step used in the calculation was typically 5-10 sec for a flux tube when outside the twin vortex and $1 \mathrm{sec}$ for when it is inside the vortex. Initially, a diurnally reproducible ionosphere was calculated using the background convection and precipitation patterns. Then, at $12 \mathrm{UT}$, the ionosphere was subjected to the additional convection and precipitation inputs associated with the twin vortex. Because the speed of the vortex structure was $3 \mathrm{~km} / \mathrm{s}$, individual plasma flux tubes were directly affected by the vortex for only a brief period of time.

Our calculations indicated that the twin vortex had its greatest effect on the ionosphere at altitudes between about $140-300 \mathrm{~km}$, with the maximum effect occurring near $200 \mathrm{~km}$. The effect was also the largest during the first 6-8 minutes after the appearance of the vortex. In response to the enhanced electric fields and particle precipitation associated with the twin vortex, several ionospheric processes were anticipated, 

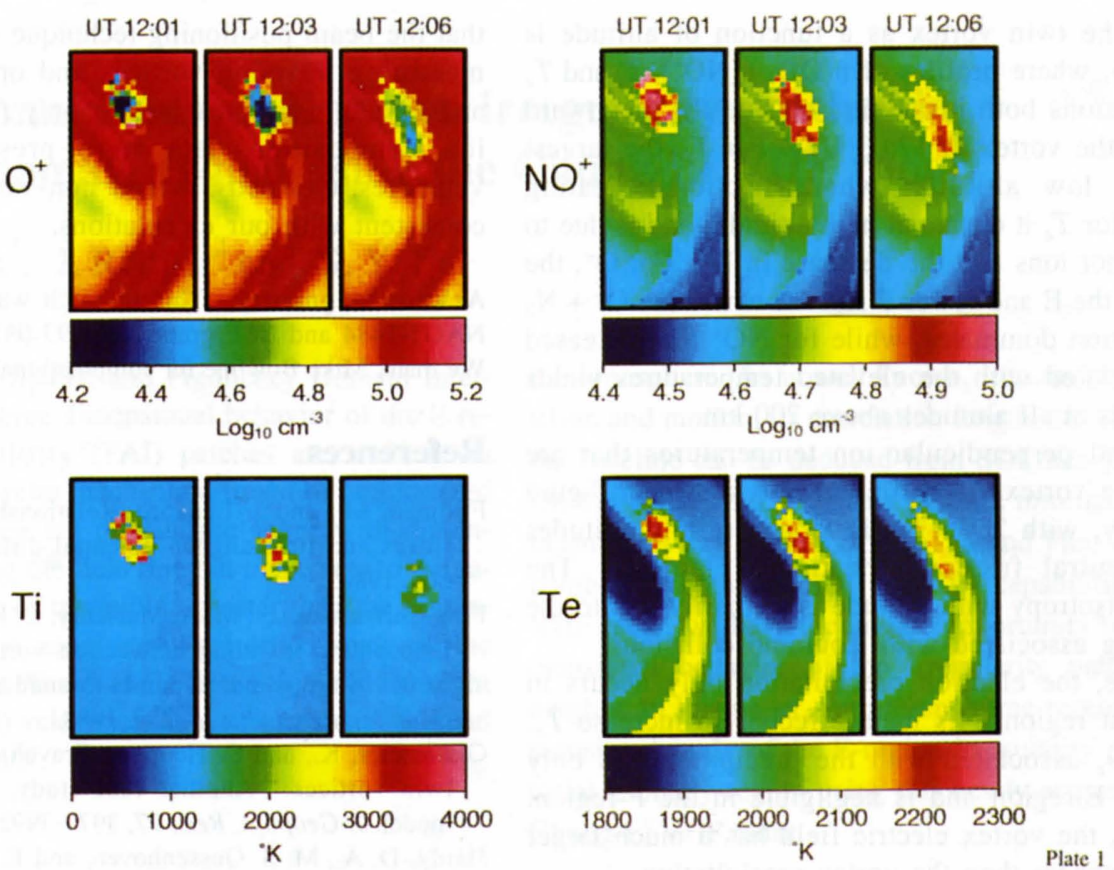

Plate 1. Snapshots of the $\mathrm{O}^{+}$and $\mathrm{NO}^{+}$densities, and the ion and electron temperatures, at $220 \mathrm{~km}$. The simulation results are for moderate solar activity and summer conditions.

included $T_{i}$ and $T_{e}$ enhancements, ion temperature anisotropies, and $\mathrm{O}^{+} \rightarrow \mathrm{NO}^{+}$composition changes [cf. Schunk et al., 1975; St.-Maurice and Schunk, 1979]. However, since the twin vortex moves fairly rapidly through the ionosphere and the different ionospheric parameters have different response times, the quantitative effect of the traveling twin vortex can only be determined via a numerical simulation.

Plate 1 shows snapshots of $\mathrm{n}\left(\mathrm{O}^{+}\right), \mathrm{n}\left(\mathrm{NO}^{+}\right), T_{i}$ and $T_{e}$ at 220 $\mathrm{km}$ and at 12:01, 12:03, and 12:06 UT for summer conditions. These parameters are only shown in the grid area of Figure 1. In response to the enhanced vortex electric fields, $T_{i}$ is initially increased to about $4000 \mathrm{~K}$ in both convection cells due to ion-neutral frictional heating. Associated with the increased $T_{i}^{\prime}$ 's is a substantial $\mathrm{O}^{+} \rightarrow \mathrm{NO}^{+}$composition change owing to the energy dependence of this reaction. Initially, $\mathrm{n}\left(\mathrm{O}^{+}\right)$is decreased by a factor of 10 and $\mathrm{n}\left(\mathrm{NO}^{+}\right)$is increased by a factor of 2.5. Like $T_{i}, T_{e}$ is also elevated in the twin vortex location, but only by about $300 \mathrm{~K}$. Unlike $T_{i}$, however, the $T_{e}$ enhancement is not symmetric. $T_{e}$ is increased due to both coupling to the hot ions and energy transfer from the precipitating electrons. The latter mechanism dominates, and since it only exists in the dayside cell (upward current region), the $T_{e}$ enhancement is asymmetric (see 12:01 UT).

As the twin vortex moves through the ionosphere, it decays and the ionosphere starts to recover after its passage. Nevertheless, the residual effect of the vortex motion can be clearly seen as streaks in the $\mathrm{n}\left(\mathrm{O}^{+}\right), \mathrm{n}\left(\mathrm{NO}^{+}\right)$and $T_{e}$ distributions, but not in the $T_{i}$ distribution. Because of the short chemical time constants at $220 \mathrm{~km}$, the density streaks are not uniform, with the largest depletions/enhancements occurring at the present location of the twin vortex. Because of the rapid $T_{i}$ response time, it is only elevated at the present location of the twin vortex. $T_{e}$ also has a rapid response time to external inputs. However, a residual streak in its distribution occurs because of the inverse relationship between $T_{e}$ and $\mathrm{N}_{\mathrm{e}}$. An $\mathrm{N}_{\mathrm{e}}$ depletion in the vortex wake yields a $T_{e}$ increase in response to the fairly uniform "background" convection and precipitation inputs.

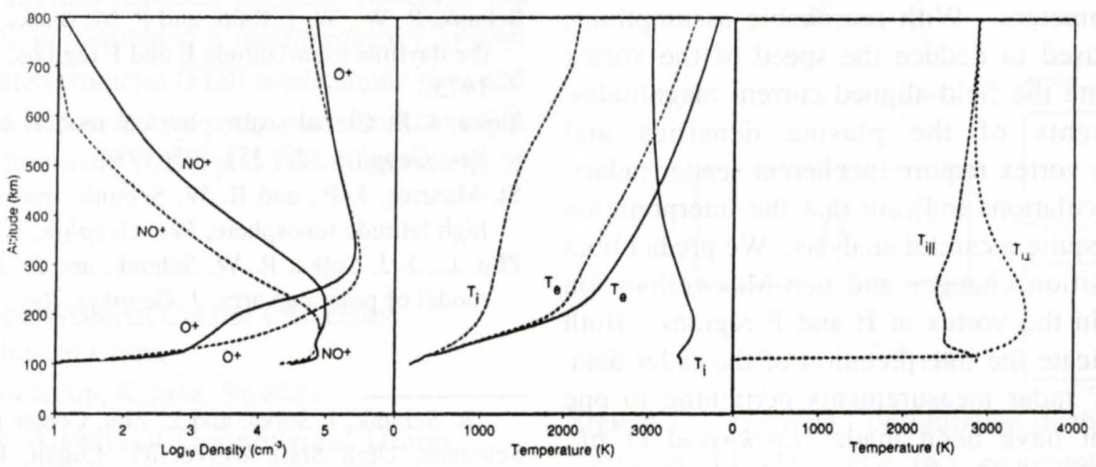

Figure 3. $\mathrm{O}^{+}$and $\mathrm{NO}^{+}$densities (left) and $\mathrm{T}_{e}$ and $\mathrm{T}_{i}$ (middle) versus altitude for locations inside (solid curves) and outside (dashed curves) the twin vortex at 12:03 UT. The right panel shows $\mathrm{T}_{i l l}$ and $\mathrm{T}_{i \perp}$ versus altitude for the location in the twin vortex. 
The effect of the twin vortex as a function of altitude is shown in Figure 3, where profiles of $\mathrm{n}\left(\mathrm{O}^{+}\right), \mathrm{n}\left(\mathrm{NO}^{+}\right), T_{i}$ and $T_{e}$ are given for locations both inside and outside the downward current region of the vortex at 12:03 UT. For $T_{i}$, the largest effect occurs at low altitudes where frictional heating dominates, while for $T_{e}$ it occurs at high altitudes and is due to heating from the hot ions and the decrease in $\mathrm{N}_{\mathrm{e}}$. For $\mathrm{O}^{+}$, the largest effect is in the $\mathrm{E}$ and lower $\mathrm{F}$ regions where the $\mathrm{O}^{+}+\mathrm{N}_{2}$ $\rightarrow \mathrm{NO}^{+}+\mathrm{N}$ reaction dominates, while for $\mathrm{NO}^{+}$the increased scale height associated with the elevated temperatures yields large enhancements at all altitudes above $200 \mathrm{~km}$.

The parallel and perpendicular ion temperatures that are associated with the vortex $T_{i}$ profile are also shown in Figure 3. The anisotropy, with $T_{i \perp}>T_{i l l}$, is largest at low altitudes where the ion-neutral frictional heating is largest. The decrease in the anisotropy with altitude is primarily due to the isotropic scattering associated with Coulomb collisions.

As noted above, the electron precipitation only occurs in the upward current region. Its main effect is to increase $T_{e}$. The increase in $\mathrm{N}_{\mathrm{e}}$ associated with the precipitation is only about $25 \%$ in the E-region and is negligible in the F-region. Hence, in general, the vortex electric field has a much larger effect on the ionosphere than the vortex precipitation.

The increased ion scale heights shown in Figure 3 are a manifestation of plasma upswelling in the twin vortex in response to the elevated $T_{e}$ and $T_{i}$. This could also lead to an enhanced polar wind, but the simulation of that flow is beyond the scope of this paper.

\section{Summary}

The transient ionospheric response to a "representative" traveling twin vortex was calculated for moderate solar activity and both summer and winter conditions. The ionospheric response was characterized by ion and electron temperature enhancements, ion temperature anisotropies, elevated plasma scale heights, $\mathrm{O}^{+}$density depletions, and $\mathrm{NO}^{+}$density increases. In general, the effects were the largest in the $\mathrm{E}$ and lower $\mathrm{F}$ regions, although the effects were evident at all altitudes. The effects in winter (not shown) were comparable to, but smaller than, the summer effects shown in this paper. The distinct ionospheric signatures associated with a twin vortex may be useful in elucidating the magnetosphericionospheric coupling processes associated with traveling twin vortices.

The ionospheric response we predict is large, but the effect is transient and may be difficult to measure. To date, by far the bulk of the twin vortex measurements have been made with ground-based magnetometers. With reasonable assumptions, such data have been used to deduce the speed of the vortex structure and to estimate the field-aligned current magnitudes. However, measurements of the plasma densities and temperatures inside the vortex require incoherent scatter radars. Unfortunately, our calculations indicate that the interpretation of the radar data will require a careful analysis. We predict both rapid $\mathrm{O}^{+} / \mathrm{NO}^{+}$composition changes and non-Maxwellian ion velocity distributions in the vortex at $\mathrm{E}$ and $\mathrm{F}$ regions. Both of these effects complicate the interpretation of the radar data. Nevertheless, EISCAT radar measurements pertaining to one traveling vortex event have been made [Lockwood et al., 1987; Lühr et al., 1993]. In the first paper, evidence of nonMaxwellian ion distributions above $300 \mathrm{~km}$ was presented at the times when the ion drift speed in the vortex exceeded the neutral thermal speed. In the second paper, the authors noted that the beam positioning technique used was not optimum for measuring traveling vortices and only line-of-sight velocities and $T_{i}$ data were presented at only four altitudes (no $T_{e}, \mathrm{~N}_{\mathrm{e}}$ or ion composition data were presented). The measured velocities, elevated $T_{i}^{\prime}$ 's and non-Maxwellian distributions are consistent with our calculations.

Acknowledgments. This research was supported by NASA grant NAG5-1484 and NSF grant ATM-93-08163 to Utah State University. We thank Mike Bowline for computational assistance.

\section{References}

Fridman, M., and J. Lemaire, Relationship between auroral electrons fluxes and field-aligned potential difference, J. Geophys. Res., 85, 664, 1980.

Friis-Christensen, E., M. A. McHenry, C. R. Clauer, and S. Vennerstrom, Ionospheric traveling convection vortices observed near the polar cleft: A triggered response to sudden changes in the solar wind, Geophys. Res. Lett., 15, 253, 1988.

Glassmeier, K., and C. Heppner, Traveling magnetospheric convection twin vortices: Another case study, global characteristics and a model, J. Geophys. Res., 97, 3977-3992, 1992.

Hardy, D. A., M. S. Gussenhoven, and E. Holeman, A statistical model of auroral electron precipitation, J. Geophys. Res., 90, 4229-4248, 1985.

Heikkila, W. J., T. S. Jorgensen, L. J. Lanzerotti, and C. G. Maclennan, A transient auroral event on the dayside, J. Geophys. Res., 94, 15291, 1989.

Heppner, J. P. and N. C. Maynard, Empirical high-latitude electric field models, J. Geophys. Res., 92, 4467-4489, 1987.

Kivelson, M. G., and D. J. Southward, Ionospheric traveling vortex generation by solar wind buffeting of the magnetosphere, $J$. Geophys. Res., 96, 1661-1667, 1991.

Lockwood, M., B. J. I. Bromage, R, B. Horne, J.-P. St.-Maurice, D. W. Willis, and S. W. H. Cowley, Non-Maxwellian ion velocity distributions observed using EISCAT, Geophys. Res. Lett., 14, 111114, 1987.

Lühr, H., W. Blawert and H. Todd, The ionospheric plasma flow and current patterns of traveling convection vortices: A case study, $J$. Atmos. Terr. Phys., 55, 1717-1727, 1993.

McHenry, M. A., C. R. Clauer, E. Friis-Christensen, P. T. Newell, and J. D. Kelly, Ground observations of magnetospheric boundary layer phenomena, J. Geophys. Res., 95, 14995, $1990 a$.

McHenry, M. A., C. R. Clauer, and E. Friis-Christensen, Relationship of solar wind parameters to continuous dayside, high latitude traveling ionospheric convection vortices, J. Geophys. Res., 95, 15007, 1990 b.

Sibeck, D. G., A model for the transient magnetospheric response to sudden solar wind dynamic pressure variations, J. Geophys. Res., 95, $3755,1990$.

Schunk, R. W., A mathematical model of the middle and high latitude ionosphere, PAGEOPH, 127, 255-303, 1988.

Schunk, R. W., W. J. Raitt, and P. M. Banks, Effect of electric fields on the daytime high-latitude $\mathrm{E}$ and $\mathrm{F}$ regions, J. Geophys. Res., 80, 3121, 1975.

Sojka, J. J., Global scale, physical models of the F region ionosphere, Rev. Geophys., 27, 371-403, 1989.

St.-Maurice, J.-P., and R. W. Schunk, Ion velocity distributions in the high latitude ionosphere, Rev. Geophys., 17, 99-134, 1979.

Zhu, L., J. J. Sojka, R. W. Schunk, and D. J. Crain, A time-dependent model of polar cap arcs, J. Geophys. Res., 98, 6139, 1993.

R. Schunk, J. Sojka, and L. Zhu, Center for Atmospheric and Space Sciences, Utah State University, Logan, UT 84322-4405 (e-mail: schunk@cc.usu.edu)

(Received: November 23, 1993; revised: March 15, 1994; accepted: April 13, 1994.) 